\title{
Clinicopathological features of gastric inflammatory myofibroblastic tumor: Report of five cases
}

\author{
YANGKUN WANG ${ }^{1}$, LAN SHEN $^{1}$, TIAN YUN ${ }^{2}$, CHAOYA ZHU ${ }^{3}$, PING WANG $^{4}$ and SUNAN WANG ${ }^{5}$ \\ ${ }^{1}$ Department of Pathology, Shenzhen Hospital of Southern Medical University, Shenzhen, Guangdong 518110; \\ ${ }^{2}$ Department of Pathology, The 989 Hospital of The Joint Logistic Support Force of The Chinese People's Liberation Army, \\ Luoyang, Henan 471031; ${ }^{3}$ Department of Pathology, The Third Affiliated Hospital, Zhengzhou University, Zhengzhou, \\ Henan 450052; ${ }^{4}$ Clinical Laboratory, BGI Genomics-Shenzhen, Shenzhen, Guangdong 518083; ${ }^{5}$ School of Electronic \\ and Communication Engineering, Shenzhen Polytechnic, Shenzhen, Guangdong 518055, P.R. China
}

Received September 10, 2019; Accepted January 8, 2021

DOI: $10.3892 /$ etm.2021.10380

\begin{abstract}
The present study reported on the histomorphological observations and immunohistochemical features of five cases of gastric inflammatory myofibroblastic tumor (IMT). Loosely arranged fat fusiform myofibroblast-fibroblasts and diffusely or patchily distributed inflammatory cells, which formed a diverse morphological structure, were observed. In the mucous vascular structure, mucoid or collagenous areas, fibromatosis- or scar-like lesions were generally $<10 \mathrm{~mm}$ in size and both had diffuse or patchy plasma cells, lymphocytes and other inflammatory-cell infiltration backgrounds. The immunophenotype was vimentin- and smooth muscle actin-positive with pan-cytokeratin, desmin and calponin expression and CD34-positive foci; furthermore, three cases were positive for anaplastic lymphoma kinase expression. Gastric IMT is rare, with unique histopathological changes and corrosion-like invasion of the smooth muscle of the stomach wall, blood vessels, nerves and adipose tissue. It should be differentiated from a variety of spindle cell tumor types and tumor-like lesions.
\end{abstract}

\section{Introduction}

Gastric inflammatory myofibroblastic tumor (IMT) is a rare and unique mesenchymal tumor type characterized as low-grade malignant or borderline tumors. In recent years, the World Health Organization (WHO) proposed the term IMT, which has gradually been recognized by experts and scholars (1-3). Gastric IMT occurs mostly in children and young adults (4). The most common sites are the mesentery, omentum, posterior peritoneum and pelvic cavity, followed

Correspondence to: Professor Sunan Wang, School of Electronic and Communication Engineering, Shenzhen Polytechnic, 4089 West Shahe Road, Xili Lake, Shenzhen, Guangdong 518055, P.R. China E-mail: wangsunan@szpt.edu.cn

Key words: gastric inflammatory myofibroblastic tumor, clinicopathology by the lungs, mediastinum and head and neck (5-7), while its occurrence in the stomach is rare. Gastric IMT mainly manifests as a nodular mass in the stomach, which resembles a malignant tumor and is easily misdiagnosed (8-10). Features of gastric IMT are normally revealed by ultrasound gastroscopy and multi-slice spiral computed tomography $(11,12)$. The clinicopathologic and immunohistochemical features of gastric IMT have been reported previously, including abdominal mass, abdominal pain, upper gastrointestinal hemorrhage; and ALK, smooth muscle actin, and vimentin staining were observed $(13,14)$. However, misdiagnosis and missed diagnosis of this disease are still common. The present study reports on the experience at our center, focusing on histopathological diagnosis and differential diagnosis with the aim to reduce the rates of missed diagnosis and misdiagnosis.

\section{Patients and methods}

Patients. The present study reports on five cases of gastric IMT, including one case encountered at the Department of Pathology of Shenzhen Hospital of Southern Medical University (Shenzhen, China), two cases from the Pathology Department of the 989 Hospital of the Joint Logistics Support Department of the Chinese People's Liberation Army (Luoyang, China), one case from the Department of Pathology of the Third Affiliated Hospital of Zhengzhou University (Zhengzhou, China) and one case was collected from the 990th Hospital of the Joint Logistics Support Force of the People's Liberation Army. These five cases of gastric IMT were definitively diagnosed between February 2015 and February 2018. Gastric IMT was diagnosed according to the histological diagnostic criteria of gastric tumor pathology (10) and the 2010 edition of the WHO digestive system tumor gastric cancer histology classification (1). Of the 5 cases of gastric IMT, 3 patients were male and 2 were female; the age ranged from 12 to 41 years and the median age was 23 years. The clinical symptoms included fatigue, epigastric fullness, abdominal pain, melena, upper gastrointestinal bleeding and obstruction after eating.

Sample preparation. Surgical specimens were fixed within $30 \mathrm{~min}$ after excision in freshly prepared $4 \%$ formaldehyde for 

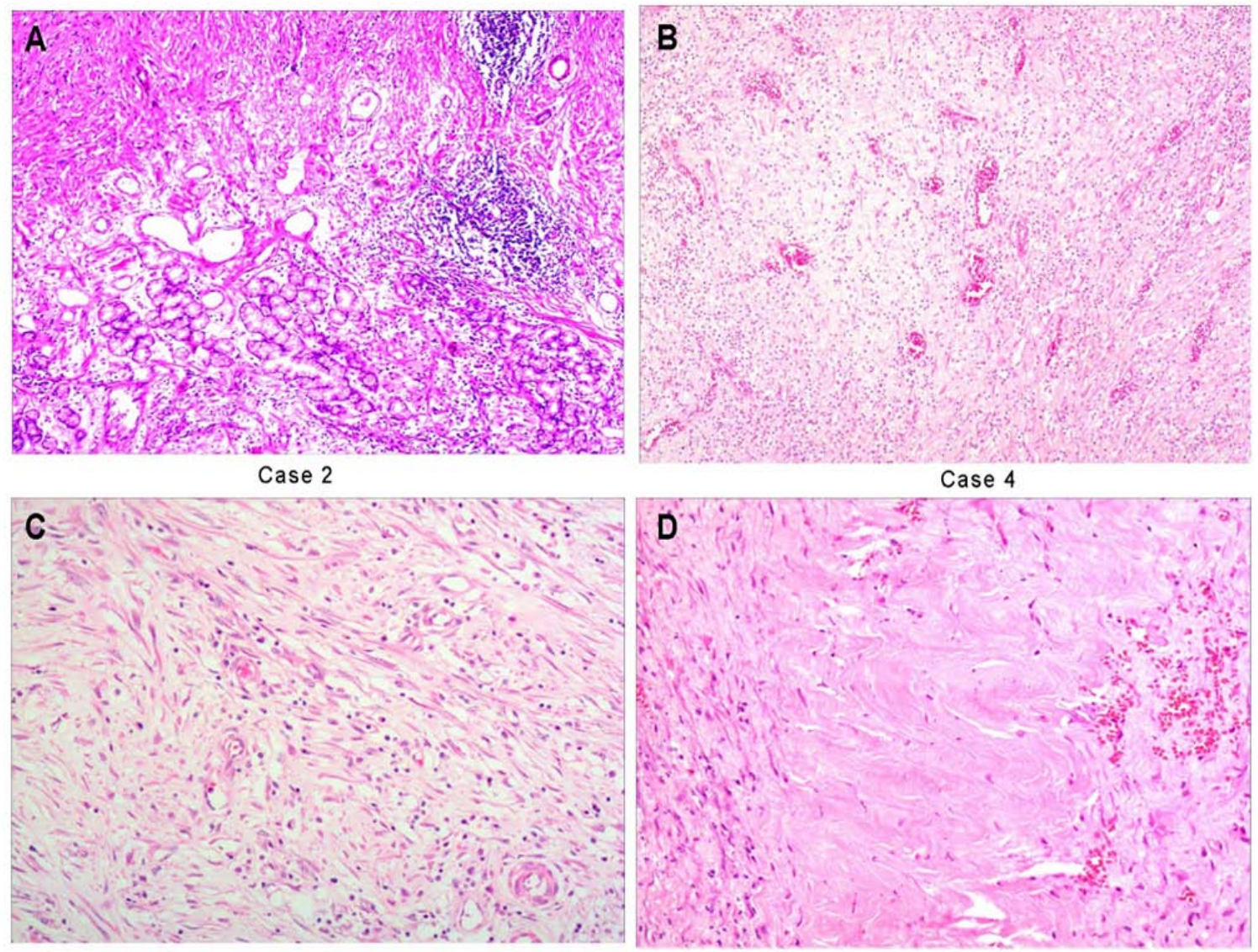

Case 3

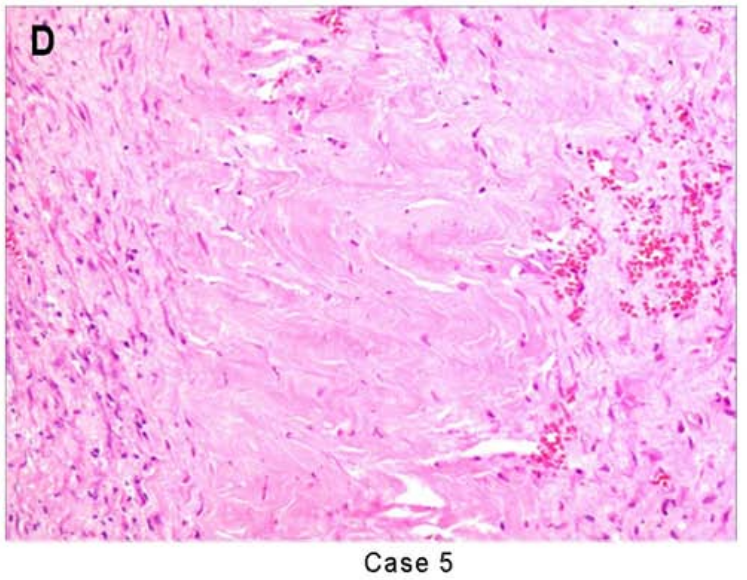

Figure 1. Histologic examination of gastric tissue samples from case 2 stained with H\&E. (A) The boundary between the mucosa of the gastric gland and the tumor was clear, but there was no capsule, a frequently staggered arrangement was present and the gastric glands frequently exhibited hyperplasia (case 2), (B) Obese or fusiform myofibroblasts are loosely arranged, with numerous vascular and plasma cells, lymphocytes and neutrophils infiltrating, forming mucous vascular structures, which is similar to granulation tissue or other reactive lesions (case 4). (C) The proliferating spindle cells are tightly arranged sarciniform and the inflammatory cells exhibit diffuse infiltration, similar to fibromatosis (case 3). (D) Regional collagenization and low cell density, similar to scar tissue (case 5) (magnification, x200 in A, C and D and x100 in B; scale bars, $50 \mu \mathrm{m}$ in A, C and D and $100 \mu \mathrm{m}$ in B).

8-48 $\mathrm{h}$ and the ratio of stationary liquid to tissue volume was 10:1. For H\&E staining and immunohistochemical examination, six to eight pieces of tumor tissue (conventional cutting of proximal end, distal margin and tumor, and adjacent gastric mucosa was not used), including the deepest infiltration point and the nearest serosal layer, were obtained.

Immunohistochemical staining. The EnVision two-step method was used for staining. Ready-to-use primary antibodies against vimentin (clone V9, Cat No. kit-0019), $\alpha$-smooth muscle actin ( $\alpha$-SMA; clone 1A4, Cat No. kit-0006), desmin (clone D33, Cat No. MAB-0766), calponin (clone MX023, Cat No. MAB-0712), anaplastic lymphoma kinase (ALK) p80 (polyclonal; Cat No. MAB-0281), CD34 (clone OBEnd/10; Cat No. kit-0004), pan-cytokeratin (CKpan; clone AE1/AE3, Cat No. kit-0009), CD117 (clone MX041, Cat No. kit-0029), anoctamin 1 (DOG1; clone SP31, Cat No. kit-0035), S-100 (clone 4C4.9, Cat No. kit-0007) and Ki-67 (clone SP6; Cat No RMA-0542) were purchased from Fuzhou Maixin Biotech Co., Ltd. Based on the requirements of the primary antibody, the corresponding antigen retrieval was performed and the steps were carried out according to the kit instruction of each antibody. For Antigen retrieval, the slides were placed in $10 \mathrm{mM}$ sodium citrate buffer ( $\mathrm{pH} \mathrm{6.0)}$ and heated samples at $95^{\circ} \mathrm{C}$ in water bath for $20 \mathrm{~min}$ followed by slowly cooling to room temperature. Positive and negative controls (Fuzhou Maixin Biotech Co., Ltd.) were established during the staining, which were included in the respective antibody kits provided by manufacturer (Fig. S1).

\section{Results}

Pathological features. The cases, including the patients' age and sex, as well as tumor size, microscopic structure and immunohistochemical staining results are summarized in Table I. In three cases, the gastric IMT was located in the gastric antrum and in two cases, it was situated in the gastric body. All tumors formed a bulging mass that protruded into the gastric cavity; the submucosa and the muscularis propria were involved in four cases and the full thickness of the stomach wall was involved in one case. The smooth surface mucosa was involved in three cases and surface mucosal ulcer formation with necrosis was present in two cases. All tumors had clear boundaries, were acapsular, nodular, had a slightly harder texture compared with the surrounding normal regions and the cut surface was soft and grayish-white mass. The tumor diameter ranged from 2.7 to $19.1 \mathrm{~cm}$ with an average tumor diameter of $5.4 \mathrm{~cm}$. The mucosal surface of the stomach 


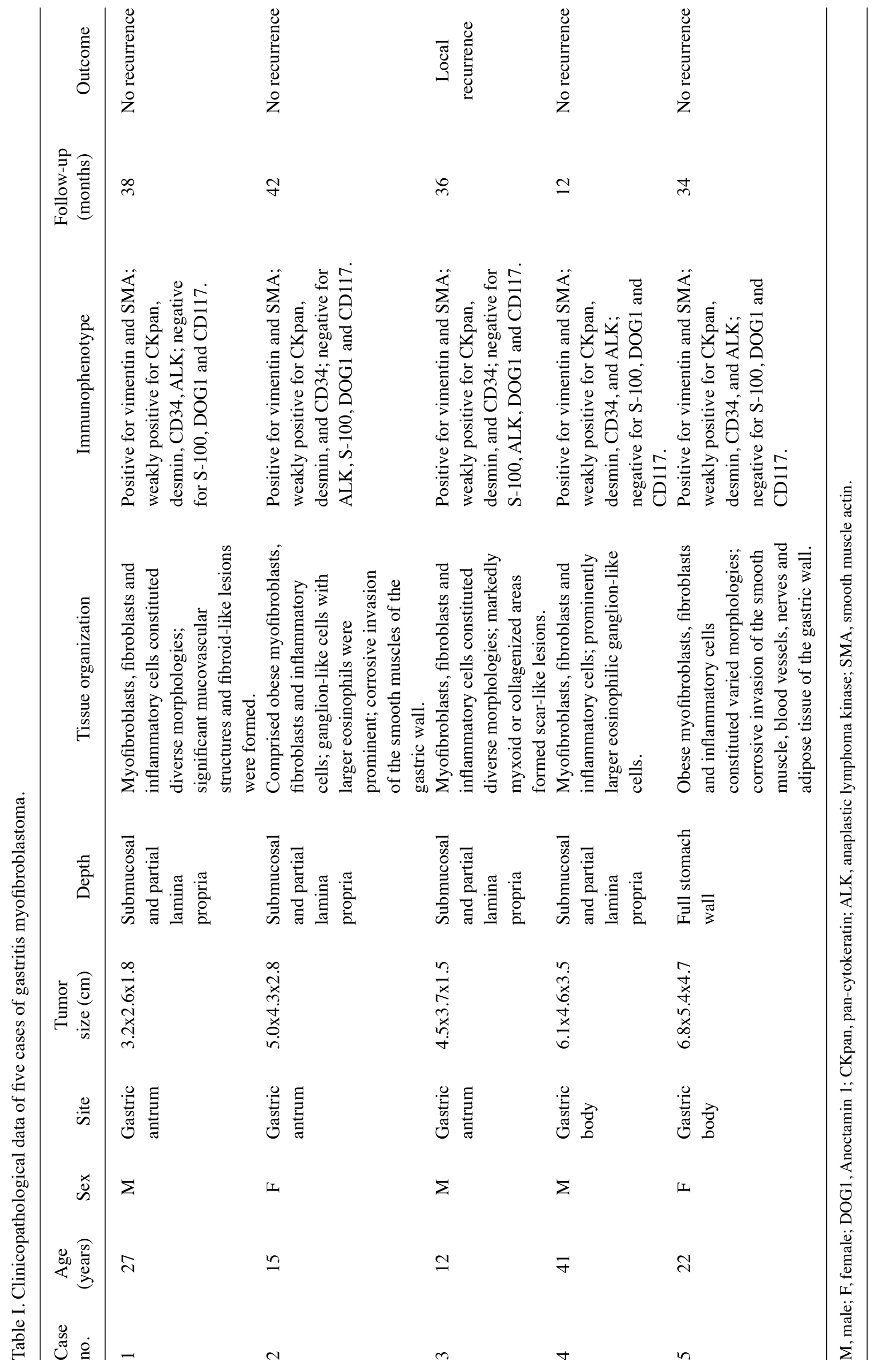



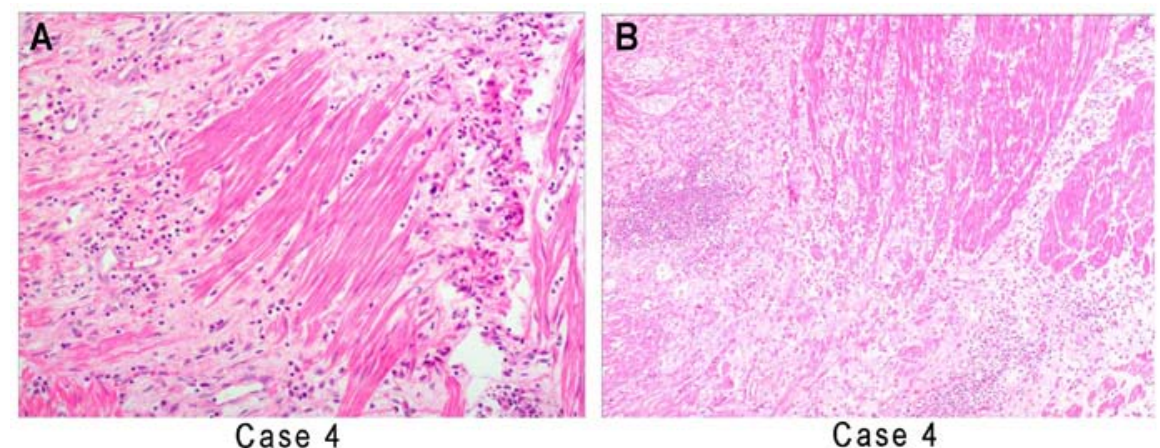

Figure 2. Infiltration in a gastric inflammatory myofibroblastic tumor (case 4). (A) Spindle-shaped myofibroblasts, fibroblasts and inflammatory cells jointly and corrosively invade the smooth muscle tissues of the stomach wall (H\&E staining; magnification, x200). (B) Corrosively disrupted smooth muscle fiber (H\&E staining; magnification, $\mathrm{x} 100)$.
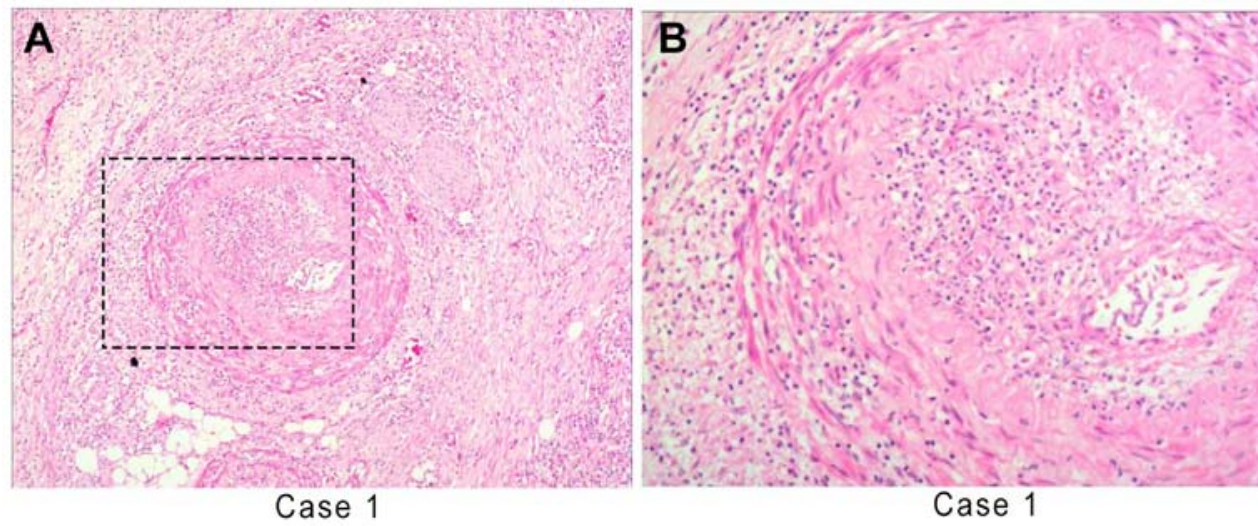

Figure 3. Infiltration in a gastric inflammatory myofibroblastic tumor (case 1). (A) Spindle-shaped myofibroblasts, fibroblasts and inflammatory cells corrosively invade the blood vessels (H\&E staining; magnification, x40; scale bar, $100 \mu \mathrm{m}$ ). (B) Magnified window displaying spindle-shaped myofibroblasts, fibroblasts and inflammatory cells in the blood vessels (H\&E staining; magnification, x100; scale bar, $50 \mu \mathrm{m}$ ).

cavity was smooth or ulcerated with necrosis (Fig. 1A). Tumors were mainly composed of spindle-shaped myofibroblasts, fibroblasts and inflammatory cells. Histomorphology indicated that the fat fusiform myofibroblast-fibroblasts were loosely arranged and the inflammatory cells were diffusely or patchily distributed. Different areas formed a diverse range of morphological structures. On the edematous mucus-like background, mucous vascular structures are formed. There was infiltration of proliferating myofibroblast-fibroblasts and numerous vascular and plasma cells, lymphocytes and neutrophils, which was similar to granulation tissue or reactive lesions (Fig. 1B). The proliferating spindle cells were tightly sarciniform, arranged with varying sizes of mucin-like or collagenous areas, which was similar to fibromatosis (Fig. 1C). In the collagenous fiber-rich area, the cell density was low and inflammatory cell components were relatively sparse, which was similar to a scar-like structure (Fig. 1D). The prominent areas of myofibroblast proliferation contained numerous ganglion-like cells. In the mucous vascular structure, mucinous or collagenous areas, i.e., fibromatosis- or scar-like lesions, were generally $<10 \mathrm{~mm}$ in size and both had diffuse or patchy plasmocytes, lymphocytes and other inflammatory cell infiltration backgrounds. Gastric IMT infiltration involved joint corrosive invasion by spindle-shaped myofibroblasts, fibroblasts and inflammatory cells of the smooth muscles of the stomach wall and adipose tissues (Fig. 2).

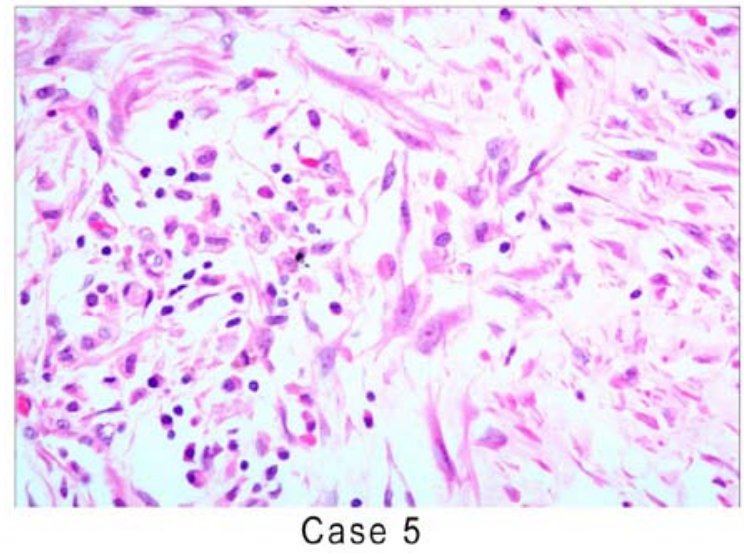

Figure 4. Myofibroblasts had fusiform and polygonal shapes (case 5), the nuclei were vacuolated and ganglion-like cells with large eosinophilic nucleoli were present (H\&E staining; magnification, x400; scale bar, $20 \mu \mathrm{m}$ ).

Corrosive invasion around the blood vessels and nerves was also able to protrude into the lumen, which was similar to infantile myofibroblastic disease (Fig. 3). Cytologically, the myofibroblast and fibroblast nuclei were fat spindle-shaped with small nucleoli and certain areas exhibited mild atypia. The myofibroblasts had fusiform and polygonal shapes and the nucleus was vacuolated, forming ganglion-like cells 

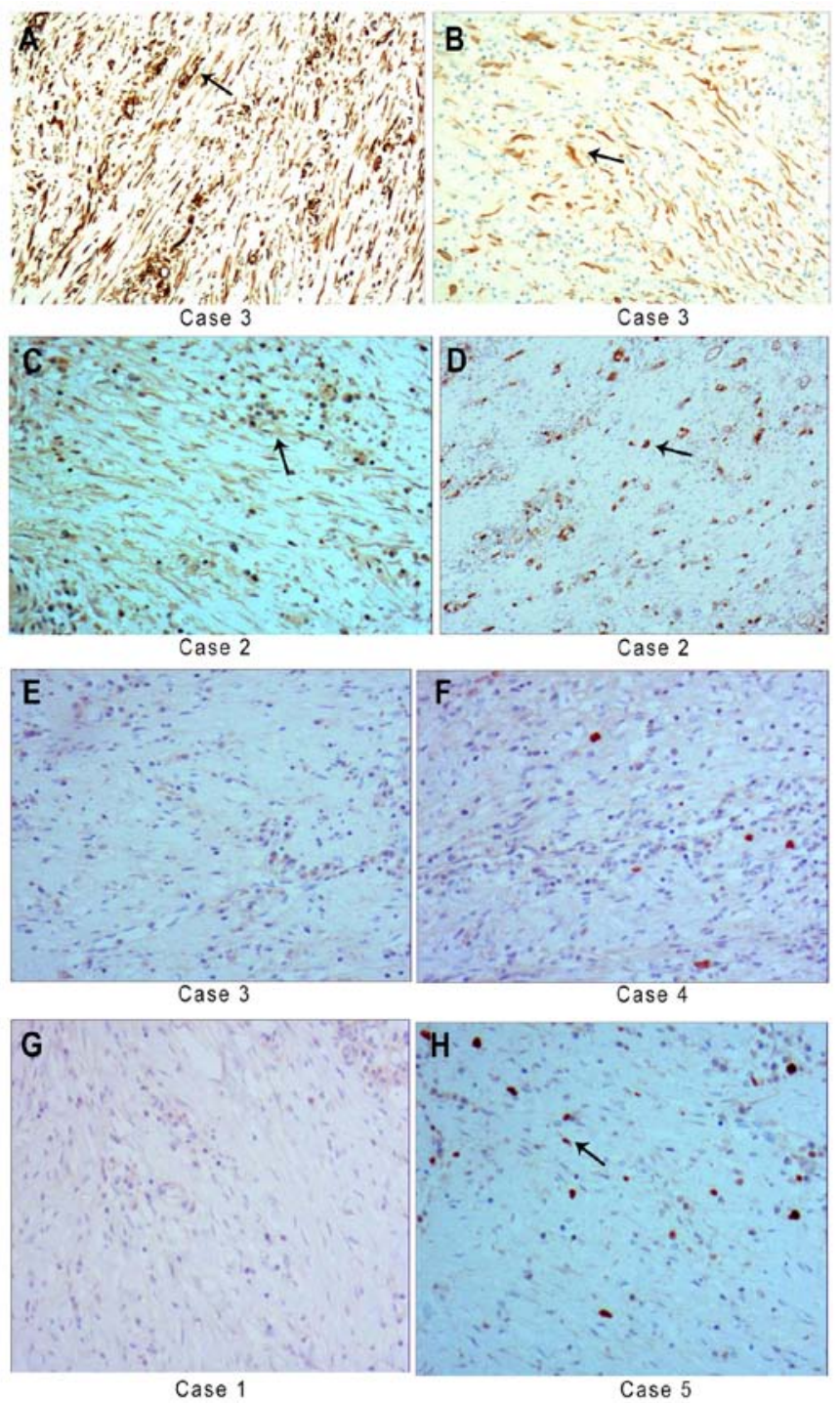

Figure 5. Gastric inflammatory myofibroblastic tumor immunophenotypes. (A) Vimentin (arrow) exhibited diffuse positive expression (case 3). (B) Pan-cytokeratin (arrow) exhibited partial positive expression (case 3). (C) Anaplastic lymphoma kinase (arrow) with focal positive expression (case 2). (D) CD34 (arrow) with focal positive expression (case 2). (E) S-100-negative expression (case 3). (F) Anoctamin 1-negative expression (case 4). (G) CD117-negative expression (case 1). (H) Ki-67-positive expression (arrow; 5-15 per 100 cells; case 5; magnification, x200; scale bar, $50 \mu \mathrm{m}$ for all).

with large eosinophilic nucleoli (Fig. 4). The inflammatory cell types included plasma cells and lymphocytes, regional neutrophils, tissue cells and multinucleated giant cells. There were 3-7 mitotic figures per 10 high-power fields.

Immunohistochemical staining. All five cases had positive but diffuse vimentin (Fig. 5A) and SMA expression and CKpan (Fig. 5B), desmin and calponin expression were partly positive. Furthermore, three cases had focal positive expression of ALK (Fig. 5C). Focal positive expression of CD34 was detected in two cases (Fig. 5D). The cases were negative for S-100 (Fig. 5E), DOG1 (Fig. 5F) and CD117 expression (Fig. 5G). $\mathrm{Ki}-67$-positive expression was present in 5-15 per 100 cells in one slide (Fig. 5H). Positive and negative control images are provided in Fig. S1.
Follow-up. The patients were followed up by telephone and the follow-up time was 12-42 months. One patient had local recurrence at the primary tumor site at 3 years and 6 months after local resection of the stomach. This patient underwent a second resection. The other four cases had no recurrence.

\section{Discussion}

IMT was first reported in two cases of benign pulmonary spindle cell tumor in 1939 (11). In 1954, it was proposed that pulmonary spindle cell proliferation was a post-inflammation tumor, which was later termed inflammatory pseudotumor and became a disease classification (15). After the 1980s, it was determined that inflammatory pseudotumor is closely related to certain actual tumors (16). The terms plasma cell granuloma, fibrinous xanthogranuloma, mucinous hamartoma, pseudosarcoma and inflammatory pseudosarcomatoid myofibroblast sarcoma have been used to describe the disease (11). Since the initial case report, numerous cases have been accumulated in recent years, which may be utilized for exploring the clinical manifestations and pathological features of IMT. The WHO soft tissue tumor pathology and genetic classification defines it as a mesenchymal tumor composed of differentiated myofibroblastic spindle cells with numerous inflammatory cells, with low-grade malignant or borderline tumor characteristics (1). The present study reported on five cases of gastric IMT and indicated that the tumor formed a bulging mass protruding into the gastric cavity, involving the stomach wall. The tumor was acapsular, nodular and the cut surface was soft and grayish-white mass. The tumor diameters ranged between 2.7 and $19.1 \mathrm{~cm}$ with an average tumor diameter of $5.4 \mathrm{~cm}$, similar to that reported in a previous study $(4.5-8 \mathrm{~cm})(13)$. However, the large amount of mature bone-like tissue reported in a case study was not observed (14). S-100, CD21, CD34, CD35, CD68 and CD117 were negative in all IMTs (13); CD117, CD34, DOG1, CK, S100, epithelial membrane antigen (EMA) and desmin were all negative in a previous case report (14). However, CD34 exhibited partial positive expression in the present study. ALK was positive in the present study, similar to the result reported in the previous case study (14).

A total of six histopathological diagnostic key points may be proposed: i) The tumor is acapsular and nodular and the cut surface is soft and grayish-white mass; ii) the fat fusiform myofibroblast-fibroblasts are loosely arranged and the inflammatory cells are diffusely or patchily distributed, forming a diverse morphological structure; iii) in the mucous vascular structure, mucinous or collagenous areas, or fibromatosis- or scar-like lesions, are generally $<10 \mathrm{~mm}$ in size and both have diffuse or patchy plasma cells, lymphocytes and other inflammatory cell infiltration backgrounds; iv) corrosive invasion of the smooth muscles of the stomach wall, blood vessels, nerves, and adipose tissues is present; v) cytologically, the myofibroblast and fibroblast nuclei are wide spindle-shaped, with small nucleoli, and certain areas exhibit mild atypia. In certain regions, myofibroblasts form fusiform and polygonal shapes and the nucleus is vacuolated, forming ganglion-like cells with large eosinophilic nucleoli; vi) immunophenotype: Positive but diffuse vimentin and SMA diffuse expression; CKpan, desmin, calponin and CD34 partial positive expression; $50 \%$ of cases are positive for ALK expression. 
The etiology of gastric IMT remains elusive and it may originate from stem cells differentiated into myofibroblasts in the gastric mesenchyme. Associated pathogenic factors include surgery, trauma, inflammation, abnormal repair or unique infection (17). Genetically, the tumors are heterogeneous; $50-70 \%$ of pediatric or young patients have clonal cytogenetic rearrangements; the affected chromosome is 2 p23 and the ALK gene on this fragment fuses with various partner genes, such as the gene for tropomyosin 3 (TPM3), TPM4, or Ran-binding protein 2, whereas these changes are not common in patients aged $>40$ years (18). The ALK fusion gene results in activation and overexpression of the tyrosine kinase domain at the C-terminus of the ALK protein, which is limited to neoplastic myofibroblasts. Immunohistochemical staining of the ALK protein C-terminus is the most effective means of determining the presence of ALK gene rearrangement in IMT. In gastric IMT, immunohistochemical staining indicated positive ALK expression in 50-60\% of cases (19). In the present study, two patients were ALK-negative and both were $>35$ years old, which is consistent with previous studies $(20,21)$.

Gastric IMT has no specific clinical manifestations, but has unique morphological features on histopathology. Therefore, the pathological diagnosis should be differentiated from benign and malignant spindle cell tumors and tumor-like lesions:

I) Inflammatory fibrous polyps, which are mesenchymal hyperplasia composed of a mixture of spindle cells, small blood vessels and inflammatory cells (particularly eosinophils). The age at onset is usually 60-75 years. The average diameter is $1.5 \mathrm{~cm}$ and it is a non-pedunculated, hard polypoid mass. Histologically, inflammatory fibrous polyps are composed of loose connective tissue; the major component is fusiform fibroblasts mixed with varying numbers of inflammatory cells and hyperplastic thin-walled blood vessels, with regional edema or mucoid background. The inflammatory cells are mainly lymphocytes and eosinophils, and at times, eosinophils are the main component of inflammation, frequently surrounding the blood vessels. Occasionally, hyperplastic mesenchymal cells surround the small blood vessels and medium-sized blood vessels to form a concentric-circular structure. Immunohistochemical staining indicates positive vimentin and CD34 expression and negative SMA and ALK expression (22).

II) Gastrointestinal stromal tumor (GIST) is the most common type of mesenchymal tumor of the stomach. It is more common in older patients with a median age of $60-65$ years. GIST may occur anywhere in the stomach, from the smallest adherent nodules to large complex masses intracavity and outside of the cavity. GIST has numerous histological types, most of which are spindle-shaped cell types. A small number of epithelial-like cell types and hybrid spindle epithelial-like cells may be present in a small proportion of cases and certain special forms have sarcomatoid characteristics, accompanied by numerous nuclear heterotypes and mitotic phases, which are pleomorphic forms. Most GISTs are positive for CD117 and DOG1 expression and certain GISTs are positive for CD34 and S-100 expression. By contrast, CD117 and DOG1 expression is negative in gastric IMT and in certain cases, ALK expression is present $(23,24)$.

III) Primary invasive fibromatosis of the stomach is composed of well-differentiated fibroblasts, with varying amounts of collagen fibers, a single cell component and a small number of inflammatory cells; strong invasion and destruction of surrounding tissues is present, with the characteristics of directional invasion and destruction of smooth muscle, blood vessels and nerve tissue. Immunohistochemical staining is positive for vimentin, SMA and $\beta$-catenin; in particular, $\beta$-catenin expression is positive in the nuclei; CKpan, CD34, S-100, desmin and ALK are not expressed (25).

IV) Malignant solitary fibrous tumor (SFT) of the stomach is composed of no fixed tissue, the histological features are irregular distribution of areas scarce and rich in tumor cells and there is denser scar-like collagen fiber deposition and branching vascular peripheral cell tumor-like vascular separation (hemangiopericytoma-like area). Gastric malignant SFT cells are abundant, tumor cells are at least moderately to severely heterotypic and there is tumor tissue necrosis. Lymph node metastasis occurs in gastric malignant SFT. The immunophenotype is positive for CD99, CD34, Bcl-2 and vimentin in tumor cells; in addition, focal weak positive expression of CKpan, EMA, SMA, S-100 and desmin is present. ALK, CD68, CD163, CD21, CD23, $\beta$-catenin, CD117 and DOG1 are not expressed (26).

V) In schwannomas, the nuclei are arranged in palisades. Immunophenotypically, S-100 expression is positive and glial fibrillary acidic protein is usually expressed, while CD117, ALK, desmin and SMA expression are negative.

VI) Synovial sarcoma, a malignant mesenchymal tumor type, exhibits varying degrees of epithelioid differentiation, with characteristic chromosomal translocation $\mathrm{t}(\mathrm{X} ; 18)$ (p11;q11), resulting in synovial sarcoma (SS)-specific, SS18-SSX gene fusion (27). The immunophenotype is CK and EMA positive expression.

In conclusion, gastric IMT is rare, with unique histopathological changes and corrosive invasion of the smooth muscle of the stomach wall, blood vessels, nerves and adipose tissue. It should be differentiated from spindle cell tumors and tumor-like lesions, where a frequent and long-term follow-up strategy should be established to improve the diagnostic rate and to reduce the rates of missed diagnosis and misdiagnosis. In the present study, no western blot or PCR analysis was used to support the immunohistochemical data, which is a limitation of the study. As $\sim 50 \%$ of conventional IMTs overexpress ALK, the ALK level detected by western blot and/or PCR analysis should aid the diagnosis of IMT, although being ALK-negative is not an indicator to exclude IMT. In the future, novel biomarkers should be developed to aid the diagnosis of this disease.

\section{Acknowledgements}

Not applicable.

\section{Funding}

No funding was received.

\section{Availability of data and materials}

All data generated or analyzed during this study are included in this published article. 


\section{Authors' contributions}

YW, SW and LS conceived the study, participated in its design and coordination, wrote the manuscript and secured funding. LS, CZ, and TY performed the experiments. LS, TY, PW, SW and $\mathrm{CZ}$ collected the data and performed the data analysis and the follow-up of the patients. YW, SW and LS checked and approved the authenticity of the raw data. All authors read and approved the final manuscript.

\section{Ethics approval and consent to participate}

Informed consent was obtained from the subjects; for minors, the guardians of the patients provided informed consent for their child to be included in this study. This study was approved by the Institutional Review Board at Shenzhen Hospital of Southern Medical University (Shenzhen, China).

\section{Patient consent for publication}

Not applicable.

\section{Competing interests}

The authors declare that they have no competing interests.

\section{References}

1. Odze RD, Lam AK, Ochiai A and Washington MK: WHO classification of tumours of the digestive system. Lyon: International Agency for Research on Cancer, 2019

2. Theilen TM, Soerensen J, Bochennek K, Becker M, Schwabe D, Rolle U, Klingebiel T and Lehrnbecher T: Crizotinib in $\mathrm{ALK}^{+}$ inflammatory myofibroblastic tumors-Current experience and future perspectives. Pediatr Blood Cancer 65: 65, 2018.

3. Raggio B and Chheda N: Inflammatory myofibroblastic tumor of the epiglottis excised with a carbon dioxide laser: Case report and literature review. Ear Nose Throat J 97: E31-E33, 2018.

4. Yutaka Y, Sato T, Matsushita K, Aiba H, Muranishi Y, Sakaguchi Y, Sugiura T, Okada M, Nakamura T and Date H: Pulmonary inflammatory myofibroblastic tumor in children: A case report and brief review of literature. Semin Thorac Cardiovasc Surg 30: 230-237, 2018

5. Kube S, Vokuhl C, Dantonello T, Scheer M, Hallmen E, Feuchtgruber S, Escherich G, Niggli F, Kuehnle I, von Kalle T, et al: Inflammatory myofibroblastic tumors-A retrospective analysis of the Cooperative Weichteilsarkom Studiengruppe. Pediatr Blood Cancer 65: e27012, 2018.

6. Hu J and Chen TB: Inflammatory myofibroblastic tumor of the paranasal sinus: Three cases report. Lin Chung Er Bi Yan Hou Tou Jing Wai Ke Za Zhi 31: 722-724, 2017 (In Chinese).

7. Sagar AES, Jimenez CA and Shannon VR: Clinical and Histopathologic correlates and management strategies for inflammatory myofibroblastic tumor of the lung. A case series and review of the literature. Med Oncol 35: 102, 2018.

8. Hayashi M, Kawakubo H, Mayanagi S, Nakamura R, Suda K, Wada N and Kitagawa Y: Gastric inflammatory myofibroblastic tumor treated with combined laparoscopic and endoscopic gastric wedge resection: A case report. World J Surg Oncol 16 : $161,2018$.

9. Jadhav M, Harvi R, Patil R and Kittur S: Inflammatory myofibroblastic tumor of the stomach presenting as an Exophytic Mass-A diagnostic dilemma. Turk Patoloji Derg 35: 151-156, 2017.
10. Wang Y: Gastric Cancer Pathology [M]//Chunfang Gao, Yangkun Wang. Digestive System Oncology. 1st Edition, Beijing, People's Military Medical Press, pp296-404, 2012.

11. Strianese D, Tranfa F, Finelli M, Iuliano A, Staibano S and Mariniello G: Inflammatory myofibroblastic tumor of the orbit: A clinico-pathological study of 25 cases. Saudi J Ophthalmol 32: 33-39, 2018.

12. Lee JE, Choi SY, Lee HK, Yi BH, Lee MH, Lee S, Lee SJ, Lee J and Jeong WK: Computed tomographic features of inflammatory myofibroblastic tumour of the stomach in adult patients: An analysis of five multicentre cases with literature review. J Med Imaging Radiat Oncol 62: 769-776, 2018.

13. Shi H, Wei L, Sun L and Guo A: Primary gastric inflammatory myofibroblastic tumor: A clinicopathologic and immunohistochemical study of 5 cases. Pathol Res Pract 206: 287-291, 2010.

14. Cheng B, Yang C, Liu Z, Liu L and Zhou L: Primary gastric inflammatory myofibroblastic tumor: A case report. Medicine (Baltimore) 97: e13423, 2018.

15. Pettinato G, Manivel JC, De Rosa N and Dehner LP: Inflammatory myofibroblastic tumor (plasma cell granuloma). Clinicopathologic study of 20 cases with immunohistochemical and ultrastructural observations. Am J Clin Pathol 94: 538-546, 1990.

16. Coindre JM: Histologic classification of soft tissue tumors (WHO, 1994). Ann Pathol 14: 426-427, 1994 (In French).

17. Lemale J, Boudjemaa S, Parmentier B, Ducou Le Pointe H, Coulomb A and Dainese L: A pseudotumoral lesion revealing Meckel's diverticulum. Arch Pediatr 23: 1157-1160, 2016 (In French)

18. Rao N, Iwenofu H, Tang B, Woyach $\mathrm{J}$ and Liebner DA: Inflammatory myofibroblastic tumor driven by novel NUMA1-ALK fusion responds to ALK inhibition. J Natl Compr Canc Netw 16: 115-121, 2018.

19. Fan J, Yang M, Huang B, Wang Z, Luo D, Zhang J, Zhang P Shi $\mathrm{H}, \mathrm{Li} \mathrm{Y}$ and Nie X: ALK expressed in a gastrointestinal stromal tumor harboring PDGFRA p. D842V mutation: A case report. Diagn Pathol 15: 8, 2020.

20. Mariño-Enríquez A, Wang WL, Roy A, Lopez-Terrada D, Lazar AJ, Fletcher CD, Coffin CM and Hornick JL: Epithelioid inflammatory myofibroblastic sarcoma: An aggressive intra-abdominal variant of infammatory myofibroblatic tumor with nuclear membrane or perinuclear ALK. Am J Surg Pathol 35: 135-144, 2011.

21. Koh J, Lee KL, Lee MS, Ahn HS and Chang MS: Gastric inverted hyperplastic polyp with inflammatory myofibroblastic tumor-like stroma, mimicking GI stromal tumor. Gastrointest Endosc 89: 433-435, 2019.

22. Klingbeil KD, Balaban A, Fertig RM, Gamret AC, Gong Y, Torres $\mathrm{C}$ and Satahoo SS: Inflammatory fibroid polyp of the gastric antrum presenting as hypovolemic shock: Case report and literature review. Intractable Rare Dis Res 6: 304-309, 2017.

23. Lacka DE and Nasierowska-Guttmejer A: Fibromatosisimmunohistochemical evaluation, differential diagnosis from gastrointestinal tumors, and other mesenchymal tumours. Prz Gastroenterol 14: 79-85, 2019.

24. Rowe SP and Fishman EK: Cinematic rendering of neurofibromatosis type I gastrointestinal stromal tumors. Radiology 291: 298, 2019

25. Wang YK, Jiang B, Yang YC, Wang SN, Li YY, Meng NL, Yuan XT, Jiang RD and Li ZG: Gastric aggressive fibromatosis: Report of a case and review of the literature. Int J Clin Exp Pathol 12: 372-377, 2019.

26. Voth E, Serio S, Gross J, Singh A, Dietz N and Nandipati K: Solitary fibrous tumor of the stomach with high-grade sarcomatous dedifferentiation. J Surg Case Rep 2018: rjy307, 2018.

27. Olsen G, Beal EW, Pfeil S and Dillhoff M: Primary gastric synovial sarcoma mimicking a gastrointestinal stromal tumor (GIST): Gastric synovial sarcoma. J Gastrointest Surg 22: 1450-1451, 2018.

This work is licensed under a Creative Commons Attribution-NonCommercial-NoDerivatives 4.0 International (CC BY-NC-ND 4.0) License. 